\title{
TEKERLEK İÇİ MOTORLU ELEKTRİKLİ BİR ARACIN AKTİF SÜSPANSIYYON SISTEMININ PID VE BULANIK MANTIK TABANLI KONTROLÜ
}

Mustafa Tayyip TOKSOY

Ahmet YILDIZ

Öz: Bu çalışmada tekerlek içi motorlu elektrikli bir aracın aktif süspansiyon sistemleri PID ve Bulanık Mantık denetleyicileri ile kontrol edilmiş ve performansları karşılaştırılmıştır. Doğrusal olmayan süspansiyon yaylarının kullanıldığı ve sürücü modeli ile birlikte 14 serbestlik derecesine sahip modelin hareket denklemleri çıkarılarak Matlab/Simulink ortamında çözdürülmüştür. Daha sonra PID ve Bulanık Mantık yöntemleri yardımı ile tam taşıt modelinde aktif süspansiyon sistemlerinin kontrolü gerçekleştirilmiş ve elde edilen sonuçları kontrolcü olmadan çıkan simülasyon sonuçları ile karşılaştırılmışıı. Çalışmada kontrolcü tasarımına yönelik Bulanık Mantık yönteminin hesaplama metodu, buna ait hata oranı ve hata oranının grafikleri, PID kontrolcü tasarımı, hesaplamaları ve sonuçları sunulmuştur. Sonuçta, her iki kontrolcü ile elde edilen verilerin kontrolcü olmadan elde edilenlere göre titreşım genliğini büyük oranda düşürdüğü ve ayrıca Bulanık Mantık ile yapılan kontrolün daha iyi sonuçlar verdiği gözlemlenmiştir.

Anahtar Kelimeler: PID, Bulanık, Mantık, Elektirkli, Araç, Süspansiyon

PID and Fuzzy Logic Based Control of the Active Suspension System of an Electric Vehicle with InWheel Motor

\begin{abstract}
In this study, active suspension systems of an electric vehicle with in-wheel motor are controlled with PID and Fuzzy Logic controllers and their performances are compared. The equations of motions are drived by using the 14 degrees of freedom model with nonlinear suspension springs and the driver model and solved in Matlab / Simulink environment. Then, control of active suspension systems in full vehicle model is performed with the help of PID and Fuzzy Logic methods and the obtained results are compared with the simulation outcomes without a controller. The calculation method of the Fuzzy Logic method for controller design, the related error rate and error rate graphs, PID controller design, calculations and results are presented. As a result, it is observed that the data obtained with both controllers decrease the vibration amplitude significantly compared to the ones obtained without the controller and also the control performed with Fuzzy Logic gives better results.
\end{abstract}

Keywords: PID, Fuzzy, Logic, Electrical, Vehicle, Suspension

\footnotetext{
* Bursa Uludağ Üniversitesi, Mühendislik Fakültesi, Otomotiv Mühendisliği Bölümü, Görükle Kampüs, 16059, Bursa, Türkiye.

İletişim Yazarı: Ahmet YILDIZ (ahmetyildiz@uludag.edu.tr)
} 


\section{GİRIȘ}

Taşıt performansı üç başlıkta incelenebilir. Bu performans ölçütleri; yoldan gelen bozucu etkilerinin sönümlenmesi, sürüş kabiliyeti ve güvenliğinin arttırılmasıdır. Böylelikle araç içindeki yolcuların bu titreşimlerden etkilenmesi en aza indirgenmeye çalışılmaktadır (Güçlü, 2005). Titreşimleri azaltmak için birçok süspansiyon sistemi tasarlanmıştır. Süspansiyon sistemleri genellikle üç ana kategoriye ayrılır: Pasif süspansiyon, yarı aktif süspansiyon ve aktif süspansiyon sistemi (Gillespie, 1996, Jazar, 2017, Cao ve diğ., 2011). Bunlardan biri olan pasif süspansiyon sistemi basitlik, yüksek güvenilirlik ve düşük maliyet nedeniyle aktif veya yarı aktif süspansiyon sistemlerine göre yaygın olarak kullanılmaktadır. Son zamanlarda, pasif süspansiyon sistemlerinin parametrelerini daha iyi hale getirmek amacıyla yoğun çalışmalar yapılmaktadır. (Yıldız, 2019a, Yıldız, 2019b) Öte yandan aktif süspansiyon sistemlerinin kullanımı yol şartlarına uyum sağlama özelliği sayesinde üreticiler için iyi bir seçenektir. Aktif süspansiyon sistemleri sürekli olarak değişen yol koşullarına uyum sağlama özelliğine sahiptir. Bu sistemler taşıtın hareketlerini sürekli olarak takip eder ve bu takip sonucunda ulaştığı değerleri sistem parametresi olarak kontrol eder. Gelişmiş sensörler ve sürekli bilgi besleyen mikroişlemciler sayesinde istenen referans değerine göre geri besleme sağlar. Değişken yol koşullarına yanıt vermek için aktif süspansiyonlar üstün yol tutuşu, üstün yol hissi ve güvenlik sunar. Aktif süspansiyon sistemleri şu bileşenlerden oluşur: Ayarlanabilir damperler ve yaylar, her tekerlekte bir dizi sensör ve her damper ile yayın üstünde bir aktüatör veya servo bulunur. Gelişmiş aktif süspansiyon sistemlerinde yol yüzeyindeki pürüzlülükler algılanır ve geri besleme ile kontrol edilerek taşıt titreşimleri azaltılır. Bu bileşenler, sistemin performansını iyileştirmek, damper sertliğini ve yay oranını ayarlamak için kullanılır (Allen, 2008).

Sürüş kalitesini iyileştirmek için aktif süspansiyon sistemlerinde birçok kontrolcü kullanılmaktadır. Bulanık kontrol, $\mathrm{H} \infty$ kontrolü, durum geri besleme yöntemi, yapay sinir ağları yöntemi ve kayar kip kontrol yöntemleri doğrusal olmayan sistemlerde uygulanan tekniklerdir (Rao ve Prahlad, 1997). Bu kontrol yöntemleri arasında, Hœ kontrol yöntemi, araç süspansiyon sisteminde yaygın olarak meydana gelen model belirsizlikleri, aktüatör zaman gecikmesi ve arızalar gibi sistem sorunlarını çözebilmektedir. Aktif süspansiyon sistemleri için hatayı tespit etmek ve kontrol ile süspansiyon sisteminin daha iyi performans göstermesini sağlamak amaciyla hata tespiti ve hataya dayanıklı kontrol yöntemleri kullanılmıştır (Sun ve diğ., 2014). Takagi ve Sugeno (1985) bulanık mantık modeline göre aktüatör gecikmesi ve hataları bulunan aktif süspansiyon sistemleri için bulanık Ho denetleyicisi tasarlanmıştır. Bilinmeyen aktüatör arızaları ve sensör arızaları olduğunda çok girişli ve çok çıkışlı doğrusal olmayan sistemler için izleme kontrol yöntemi önermiştir. Aktüatörlerin pnömatik ve hidrolik özellikleri nedeniyle birçok kontrol sistemi uygulamasında meydana gelen aktüatör gecikmesi, kontrolör tasarımında dikkate alınmazsa kontrol performansını düşürebilir. Bunun neticesinde ortaya çıkan sonuçlar kontrol sistemlerinde kararsızlığa neden olabilir. Sistem sürtünmesi, sistem belirsizlikleri ve harici titreşimler gibi dış bozucu etkilerin kontrol sistemi performansı üzerinde olumsuz etkisi vardır. Dış bozuklukların etkisini tahmin etmek ve telafi etmek için bozulmaları takip eden kontrol yöntemleri kullanılabilir. Bununla birlikte, süspansiyon sistemlerinin gerekli durum değişkenlerinden bazıları ölçülemediğinde, yukarıda bahsedilen yöntemleri kullanmak mümkün değildir. Bu boşluğu gidermek için geri beslemenin çıkışına bağlı kontrol şemaları geliştirilmiştir. Aktif süspansiyon sistemi için dinamik geri beslemeli $\mathrm{H} \infty$ kontrolörü önerilmiştir ( $\mathrm{Li}$ ve diğ., 2014). Ayrıca taşıt titreşimlerinin azaltılması için süspansiyon sistemlerinin analizine yönelik optimizasyon yöntemleri bulunmaktadır (Doğan ve diğ., 2015, Özkop ve Altaş, 2007, Shirahatt ve diğ., 2008). Bu çalışmalarda farklı optimizasyon teknikleri kullanılarak süspansiyon parametreleri sürüş konforunu arttırmak amacıyla optimize edilmiştir. (Badran ve diğ., 2012). Diğer bir çalışmada koltuk üzerine insan vücudu eklenmiş ve titreşimi minimize etmeye yönelik yöntemler araç içerisindekileri de dikkate alarak yapılmıştır (Yokoyama ve diğ., 2001). Öte yandan, sürüş konforunu artırmak için farklı optimizasyon yöntemleri de kullanılmaktadır. 
Mehdizadeh (2015) parçacık sürü optimizasyonu, arı kolonisi algoritması ve evrim stratejisi yöntemlerini kullanılarak bir optimizasyon gerçekleştirdi. Bu çalışmada, taşıtın süspansiyonu için optimize edilmiş parametreler, iletilen titreşimde önemli bir azalma ve sürüş konforunda da iyileşme sağlamıştır.

Son yıllarda elektrikli araçlar hızla geliştirilmekte ve otomobil endüstrisi bu yöne doğru kaymaktadır. Tekerlek içine yerleştirilecek olan motorun ağırlığı ve hacmi önemli çalışma konuları arasındadır. Elektrikli araçlarda tekerlek içine yerleştirilen motorun ağırlığını ve hacmini azaltmak için çalışmalar yapılmıştır. Nikam ve diğ. (2012) nihai sargı etkilerini en aza indirgemek ve motor ağırlığını azaltmak için yoğunlaştırılmış sargı yöntemini kullanarak mıknatıslı firçasız bir DC motor tasarladı. Takahashi ve diğg. (2017) kalıcı mıknatıs esaslı, düşük maliyetli tekerlek içi eksenel boşluklu motor tasarımı sundular. Boyutu ve ağırlı̆̆ daha da azaltmak için, yarı kapalı bir yuva yapısı yerine açık bir yuva yapısı kullanmıştır. Deneysel sonuçlar, yarı kapalı yuva yapısının boyut ve ağırlıkta azalma sağlamak için daha etkili olduğunu göstermiştir. Tekerlek içi motorlar dinamik sönümleyici olarak da tasarlanabilir. Bir tekerlek içi motorun dikey yönde titreşimi arttırmak gibi araç konforuna olumsuz etkileri vardır ve bunları azaltmanın yöntemleri mevcuttur. Bununla ilgili Oliveira ve diğ. (2017) düşük titreşim genliği gereksinimlerini karşılamak için belirli bir aralıkta sürekli olarak ayarlanabilen bir tekerlek içi yarı aktif süspansiyon tasarladı. Jin ve diğ. (2016) tekerlek içi motorlu araçların titreşim genliğinin yükseldiği ve bunu azaltmak için PID denetleye dayalı bir manyeto-reolojik (MR) yarı aktif süspansiyon sistemi tasarlamıştır. Tasarlanan sistemin, aracın sürüş konforunu arttırdığ1 simülasyon sonuçları ile gösterilmiştir.

Yukarıda bahsedilen çalışmalardan farklı olarak, bu çalışmada on dört serbestlik dereceli ve doğrusal olmayan yay karakteristiğine sahip bir tam taşıt ve insan modelinin PID ve bulanık mantık tabanlı kontrolü karşılaştırmalı olarak incelenmiştir. Modelde tam taşıta ait yedi serbestlik derecesine ilave olarak, koltuk modeliyle birlikte dört serbestlik dereceli sürücü modeli ve iki serbestlik dereceli tekerlek içi motor esas alınmıştır. Aktif süspansiyon sisteminde bulanık mantık yönteminde kullanılan hata ve hatanın türevine karşılık gelen üyelik fonksiyonları belirlenmiştir ve bunlar kullanılarak titreşim genliği azaltılmıştır. Ayrıca PID kontrolcü tasarımı yapılmış ve simülasyon sonuçları sunulmuştur. Çalışmaların sonucunda, her iki kontrolcü ile elde edilen verilerin kontrolcü olmadan elde edilen grafiklere göre titreşim genliğini büyük oranda düşürdüğü ve ayrıca Bulanık Mantık ile yapılan kontrolün daha iyi sonuçlar verdiği, PID yöntemine göre referans değerine genelde daha erken ulaştığı gözlenmiştir.

\section{DOĞRUSAL OLMAYAN TAM TAŞIT ve SÜRÜCÜ MODELI}

Tam taşıt modeli on dört serbestlik derecesine sahiptir: $x_{w f}, x_{w f r}, x_{w r r}, x_{w r l}, x_{v}, x_{p}, \theta, \emptyset, x_{i l}, x_{i r}$, $x_{t}, x_{l}, x_{u}$ ve $x_{h}$. Bunlar sırasıyla sağ ön aksın hareketini, sol ön aksın hareketini, sağ arka aksın hareketini, sol arka aksın hareketini, yolcu koltuğunun sıçrama hareketini, araç gövdesinin sıçrama hareketini, taşıt gövdesinin kendi enine ekseni etrafinda dönme (başvurma) hareketini, taşıt gövdesinin yalpa hareketini, sol arka tekerlek içi motor, sağ arka tekerlek içi motor, sürücünün baldır, uyluk, gövde ve baş kısmının deplasmanlarını göstermektedir.

Tam taşıt modelinin süspansiyon yer değiştirmeleri aşağıda verilen denklemler ile tanımlanır:

$$
\begin{aligned}
& x_{s f r t}=x_{w f r}-x_{v}+L_{f} \theta+b_{r} \emptyset \\
& x_{s f l t}=x_{w f l}-x_{v}+L_{f} \theta-b_{l} \varnothing
\end{aligned}
$$


Toksoy M.T., Yıldız A. : Tekerlek içi Mot. Elek. Arac. Akt. Süs. Sist. PID Bulanık Mant. Tab. Kontr.

$$
\begin{gathered}
x_{s r r t}=x_{w r r}-x_{v}-L_{r} \theta+b_{r} \emptyset \\
x_{s r l t}=x_{w r l}-x_{v}-L_{r} \theta-b_{l} \varnothing \\
x_{p t}=x_{v}-x_{p}-n \theta+m \emptyset
\end{gathered}
$$

Denklem 1-5 ile verilen yer değiştirmelerin türevleri ise aşağıdaki gibidir:

$$
\begin{gathered}
\dot{x}_{s f r t}=\dot{x}_{w f r}-\dot{x}_{v}+L_{f} \dot{\theta}+b_{r} \dot{\varnothing} \\
\dot{x}_{s f l t}=\dot{x}_{w f l}-\dot{x}_{v}+L_{f} \dot{\theta}-b_{l} \dot{\varnothing} \\
\dot{x}_{s r r t}=\dot{x}_{w r r}-\dot{x}_{v}-L_{r} \dot{\theta}+b_{r} \dot{\varnothing} \\
\dot{x}_{s r l t}=\dot{x}_{w r l}-\dot{x}_{v}-L_{r} \dot{\theta}-b_{l} \dot{\varnothing} \\
\dot{x}_{p t}=\dot{x}_{v}-\dot{x}_{p}-n \dot{\theta}+m \dot{\varnothing}
\end{gathered}
$$

Yukarıdaki denklemlerde $b_{l}$ ve $b_{r}$ ağırlık merkezinin sola ve sağa olan mesafesi iken, m ve n koltuk bağlantı noktasının ağırlık merkezi eksenlerine olan uzaklıklarını göstermektedir.

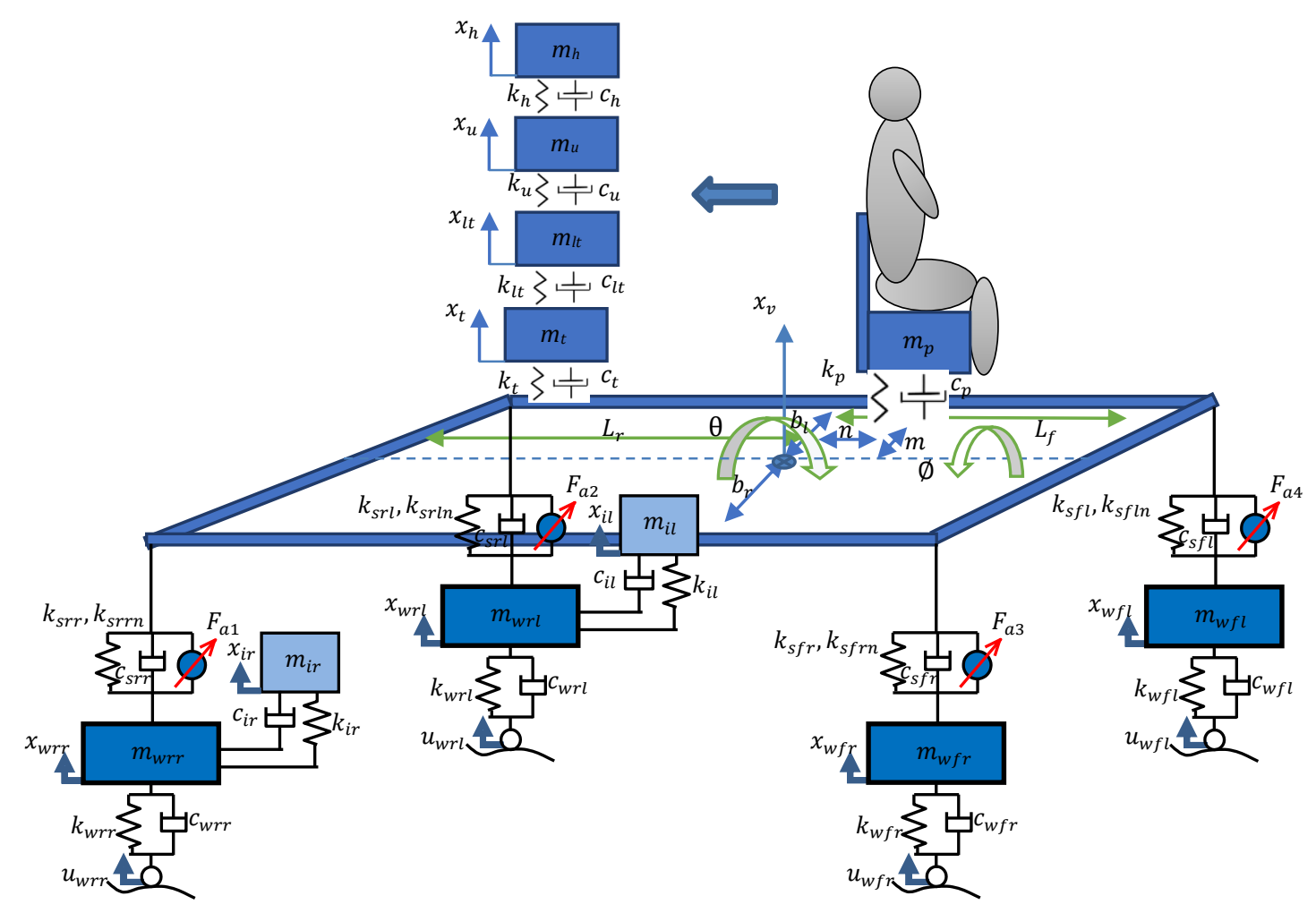

Şekil 1:

Doğrusal Olmayan Tekerlek İ̧̧i Motorlu Elektrikli Aracın Tam Taşıt ve Sürücü Modeli 
Ön sağ, ön sol, arka sağ ve arka sol süspansiyon kuvvetleri aşağıdaki gibidir:

$$
\begin{gathered}
F_{s f r}=k_{s f r}\left(x_{s f r t}\right)+k_{s f r n}\left(x_{s f r t}\right)^{3}+c_{s f r} \dot{x}_{s f r t} \\
F_{s f l}=k_{s f l}\left(x_{s f l t}\right)+k_{s f l n}\left(x_{s f l t}\right)^{3}+c_{s f l} \dot{x}_{s f l t} \\
F_{s r r}=k_{s r r}\left(x_{s r r t}\right)+k_{s r r n}\left(x_{s r r t}\right)^{3}+c_{s r r} \dot{x}_{s r r t} \\
F_{s r l}=k_{s r l}\left(x_{s r l t}\right)+k_{s r l n}\left(x_{s r l t}\right)^{3}+c_{s r l} \dot{x}_{s r l t}
\end{gathered}
$$

Benzer şekilde sürücü koltuğu süspansiyon sistemi kuvveti ile ön sağ, ön sol, arka sağ ve arka sol tekerlek kuvvetleri aşağıdaki gibi ifade edilebilirler.

$$
\begin{gathered}
F_{p}=k_{p}\left(x_{p t}\right)+k_{p n}\left(x_{p t}\right)^{3}+c_{p} \dot{x}_{p t} \\
F_{w f r}=k_{w f r}\left(u_{w f r}-x_{w f r}\right)+c_{w f r}\left(\dot{u}_{w f r}-\dot{x}_{w f r}\right) \\
F_{w f l}=k_{w f l}\left(u_{w f l}-x_{w f l}\right)+c_{w f l}\left(\dot{u}_{w f l}-\dot{x}_{w f l}\right) \\
F_{w r r}=k_{w r r}\left(u_{w r r}-x_{w r r}\right)+c_{w r r}\left(\dot{u}_{w r r}-\dot{x}_{w r r}\right) \\
F_{w r l}=k_{w r l}\left(u_{w r l}-x_{w r l}\right)+c_{w r l}\left(\dot{u}_{w r l}-\dot{x}_{w r l}\right) \\
F_{i r}=k_{i r}\left(x_{w r r}-x_{i r}\right)+c_{i r}\left(\dot{x}_{w r r}-\dot{x}_{i r}\right) \\
F_{i l}=k_{i l}\left(x_{w r l}-x_{i l}\right)+c_{i l}\left(\dot{x}_{w r l}-\dot{x}_{i l}\right)
\end{gathered}
$$

Yukarıdaki denklemlerde $k$ ve $c$ süspansiyon katılık ve sönüm katsayılarını, $m$ sembolü ise kütlelere işaret eder. Alt indisler $w f r$ ve $w f l$ sağ ön tekerleri, $w r r$ ve $w r l$ sol ön tekerleri ve $x_{w f r}$, $x_{w f l}, x_{w r r}, x_{w r l}$ ise bunlara ait yer değiştirmeleri göstermektedir.

$$
\begin{gathered}
m_{w f r} \ddot{x}_{w f r}\left(F_{w f r}-F_{s f r}-F_{a 3}\right) \\
m_{w f l} \ddot{x}_{w f l}\left(F_{w f l}-F_{s f l}-F_{a 4}\right) \\
m_{w r r} \ddot{x}_{w r r}=\left(F_{w r r}-F_{i r}-F_{s r r}-F_{a 1}\right) \\
m_{w r l} \ddot{x}_{w r l}=\left(F_{w r l}-F_{i l}-F_{s r l}-F_{a 2}\right) \\
m_{i r} \ddot{x}_{i r=}\left(F_{i r}\right)
\end{gathered}
$$


Toksoy M.T., Yıldız A. : Tekerlek içi Mot. Elek. Arac. Akt. Süs. Sist. PID Bulanık Mant. Tab. Kontr.

$$
\begin{gathered}
m_{i l} \ddot{x}_{i l=}\left(F_{i l}\right) \\
m_{v} \ddot{x}_{v}=\left(-F_{p}+F_{s f r}+F_{s f l}+F_{s r r}+F_{s r l}+F_{a 1}+F_{a 2}+F_{a 3}+F_{a 4}\right) \\
m_{p} \ddot{x}_{p}=\left(F_{p}\right)-c_{t}\left(\dot{x}_{p}-\dot{x}_{t}\right)-k_{t}\left(\dot{x}_{p}-x_{t}\right) \\
I_{v} \ddot{\theta}=\left(\left(F_{p} n\right)+\left(-F_{s f r}-F_{a 3}\right) L_{f}+\left(-F_{s f l}-F_{a 4}\right) L_{f}+\left(F_{s r r}+F_{a 1}\right) L_{r}\right. \\
\left.+\left(F_{s r l}+F_{a 2}\right) L_{r}\right) \\
I_{y} \ddot{\emptyset}=\left(\left(-F_{p} m\right)+\left(-F_{s f r}-F_{a 3}\right) b_{r}+\left(-F_{s r r}-F_{a 1}\right) b_{r}+\left(F_{s f l}+F_{a 4}\right) b_{l}+\left(F_{s r l}\right.\right. \\
\left.\left.+F_{a 2}\right) b_{l}\right) \\
m_{h} \ddot{x}_{h}=\left(c_{h}\left(\dot{x}_{u}-\dot{x}_{h}\right)+k_{h}\left(x_{u}-x_{h}\right)\right) \\
m_{u} \ddot{x}_{u}=\left(c_{u}\left(\dot{x}_{l t}-\dot{x}_{u}\right)+k_{u}\left(x_{l t}-x_{u}\right)\right)-c_{h}\left(\dot{x}_{u}-\dot{x}_{h}\right)-k_{h}\left(x_{u}-x_{h}\right) \\
m_{l t} \ddot{x}_{l t}=\left(c_{l t}\left(\dot{x}_{t}-\dot{x}_{l t}\right)+k_{l t}\left(x_{t}-x_{l t}\right)\right)-c_{u}\left(\dot{x}_{l t}-\dot{x}_{h}\right)-k_{u}\left(x_{l t}-x_{h}\right) \\
m_{t} \ddot{x}_{t}=\left(c_{t}\left(\dot{x}_{p}-\dot{x}_{t}\right)+k_{t}\left(x_{p}-x_{t}\right)\right)-c_{l t}\left(\dot{x}_{t}-\dot{x}_{l t}\right)-k_{l t}\left(x_{t}-x_{l t}\right)
\end{gathered}
$$

Dışarıdan yol girdisi $\left(u_{w f l}, u_{w f r}, u_{w r r}, u_{w r l}\right)$ olarak ön tekerlere $0,075 \sin (w t)$, arka tekerlere $0,075 \sin (w(t-s s))$ verilmiştir. Araç hızı $V=22,22 \mathrm{~m} / \mathrm{sn}$, aracın aldığ 1 yol ise $\delta=11,11 \mathrm{~m}$ olarak alınmıştır. Frekans olarak verilen w değeri $(2 \pi V / \delta)$ olarak hesaplanmış ve yaklaşık olarak 6.28 $\mathrm{rad} / \mathrm{sn}$ bulunmuştur. ss (ön ve arka tekerlekler arası gecikme) değeri aracın akslar arası mesafesi $\left(L_{f}+L_{r}\right)$ ve aracın hızına bağımlı olarak $s s=(L f+L r) / V$ şeklinde formülize edilmiş, sonucunda ss değeri bu çalışmadaki değerler için 0,1350 sn olarak bulunmuştur. Alt indisle verilen değerler dört tekere ait bilgileri göstermekte ve bunlar Şekil 1'de görülmektedir. Ayrıca yay katılığ 1 $k_{s}\left(x_{s}\right)+k_{s}\left(x_{s}\right)^{3}$ şeklinde yer değiştirmenin küpüyle orantılı doğrusal olmayan bir terimi de içermektedir. Tanımlanan bu kuvvetler yardımıyla 14 serbestlik dereceli doğrusal olmayan sistemin denklemleri aşağıdaki gibi bulunurlar:

Tam taşıt ve sürücü modeline ait değerler Tablo 1'de verilmiştir. 
Tablo 1. Tam taşıt modeline ait değerler

\begin{tabular}{|c|c|c|c|c|c|c|c|}
\hline Parametre & Değeri & Parametre & Değeri & Parametre & Değeri & Parametre & Değeri \\
\hline$m_{w f r}[\mathrm{~kg}]$ & 60 & $b_{r}$ & 0,8 & $\begin{array}{c}k_{w f r} \\
{[\mathrm{kN} / \mathrm{m}]}\end{array}$ & 190 & $\begin{array}{c}c_{w r r} \\
{[\mathrm{Ns} / \mathrm{m}]}\end{array}$ & 10 \\
\hline$m_{w f l}[\mathrm{~kg}]$ & 60 & $b_{l}$ & 0,8 & $\begin{array}{c}k_{w f l} \\
{[\mathrm{kN} / \mathrm{m}]}\end{array}$ & 190 & $\begin{array}{c}c_{w r l} \\
{[\mathrm{Ns} / \mathrm{m}]}\end{array}$ & 10 \\
\hline$m_{v}[\mathrm{~kg}]$ & 1460 & $I_{v}\left[\mathrm{kgm}^{2}\right]$ & 1450 & $\begin{array}{c}k_{w r r} \\
{[\mathrm{kN} / \mathrm{m}]}\end{array}$ & 190 & $c_{p}[\mathrm{Ns} / \mathrm{m}]$ & 800 \\
\hline$m_{p}[\mathrm{~kg}]$ & 100 & $I_{y}\left[\mathrm{kgm}^{2}\right]$ & 311 & $\begin{array}{c}k_{\text {wrl }} \\
{[\mathrm{kN} / \mathrm{m}]}\end{array}$ & 190 & $\begin{array}{c}c_{s f r} \\
{[\mathrm{Ns} / \mathrm{m}]}\end{array}$ & 2012 \\
\hline$m_{t}[\mathrm{~kg}]$ & 36 & $k_{t}[\mathrm{kN} / \mathrm{m}]$ & 20 & $k_{p}[\mathrm{kN} / \mathrm{m}]$ & 10 & $\begin{array}{c}c_{s f l} \\
{[\mathrm{Ns} / \mathrm{m}]}\end{array}$ & 2012 \\
\hline$m_{l t}[\mathrm{~kg}]$ & 5,5 & $k_{l t}[\mathrm{kN} / \mathrm{m}]$ & 144 & $\begin{array}{c}k_{p n} \\
{[\mathrm{kN} / \mathrm{m}]}\end{array}$ & 1000 & $\begin{array}{c}c_{s r r} \\
{[\mathrm{Ns} / \mathrm{m}]}\end{array}$ & 1848 \\
\hline$m_{u}[\mathrm{~kg}]$ & 15 & $k_{u}[\mathrm{kN} / \mathrm{m}]$ & 10 & $\begin{array}{c}k_{s f r} \\
{[\mathrm{kN} / \mathrm{m}]}\end{array}$ & 78,15 & $c_{s r l}[\mathrm{Ns} / \mathrm{m}]$ & 1848 \\
\hline$m_{h}[\mathrm{~kg}]$ & 4,17 & $k_{h}[\mathrm{kN} / \mathrm{m}]$ & 166,9 & $\begin{array}{c}k_{\text {sfrn }} \\
{[\mathrm{kN} / \mathrm{m}]}\end{array}$ & 0,5 & $c_{t}[\mathrm{Ns} / \mathrm{m}]$ & 330 \\
\hline$m_{w r r}[\mathrm{~kg}]$ & 70 & $\begin{array}{c}k_{\text {srr }} \\
{[\mathrm{kN} / \mathrm{m}]}\end{array}$ & 41,73 & $\begin{array}{c}k_{s f l} \\
{[\mathrm{kN} / \mathrm{m}]}\end{array}$ & 78,15 & $c_{l t}[\mathrm{Ns} / \mathrm{m}]$ & 909,1 \\
\hline$m_{w r l}[\mathrm{~kg}]$ & 70 & $\begin{array}{c}k_{\text {srrn }} \\
{[\mathrm{kN} / \mathrm{m}]}\end{array}$ & 0,01 & $\begin{array}{c}k_{\text {sfln }} \\
{[\mathrm{kN} / \mathrm{m}]}\end{array}$ & 0,5 & $c_{u}[\mathrm{Ns} / \mathrm{m}]$ & 200 \\
\hline $\mathrm{L}_{\mathrm{f}}$ & 1,5 & $\begin{array}{c}k_{s r l} \\
{[\mathrm{kN} / \mathrm{m}]}\end{array}$ & 41,73 & $\begin{array}{c}c_{w f r} \\
{[\mathrm{Ns} / \mathrm{m}]}\end{array}$ & 10 & $c_{h}[\mathrm{Ns} / \mathrm{m}]$ & 310 \\
\hline $\mathrm{L}_{\mathrm{r}}$ & 1,5 & $\begin{array}{c}k_{\text {srln }} \\
{[\mathrm{kN} / \mathrm{m}]}\end{array}$ & 0,01 & $\begin{array}{c}c_{w f l} \\
{[\mathrm{Ns} / \mathrm{m}]}\end{array}$ & 10 & $c_{i r}[\mathrm{Ns} / \mathrm{m}]$ & 1686 \\
\hline$m_{i r}[\mathrm{~kg}]$ & 30 & $k_{i r}[\mathrm{kN} / \mathrm{m}]$ & 25 & $k_{i l}[\mathrm{kN} / \mathrm{m}]$ & 25 & $c_{i l}[\mathrm{Ns} / \mathrm{m}]$ & 1686 \\
\hline$m_{i l}[\mathrm{~kg}]$ & 30 & $\mathrm{~m}[\mathrm{~m}]$ & 0,234 & $\mathrm{n}[\mathrm{m}]$ & 0,375 & & \\
\hline
\end{tabular}

\section{KONTROLCÜ TASARIMI}

\subsection{Bulanık Mantık Tabanlı Kontrol}

Bulanık Mantık sistemi dört ana parçadan oluşur: bulanıklaştırma birimi, bilgi tabanı, karar üretme mantığı ve durulaştırma birimidir. Tam taşıt modelinin kararlı halde aktif süspansiyon 
kontrolü için Bulanık Mantık denetleyicisi hata oranı (e) ile hata oranının türevi (de/dt) olarak Şekil 2'deki gibi tasarlanmıştır.
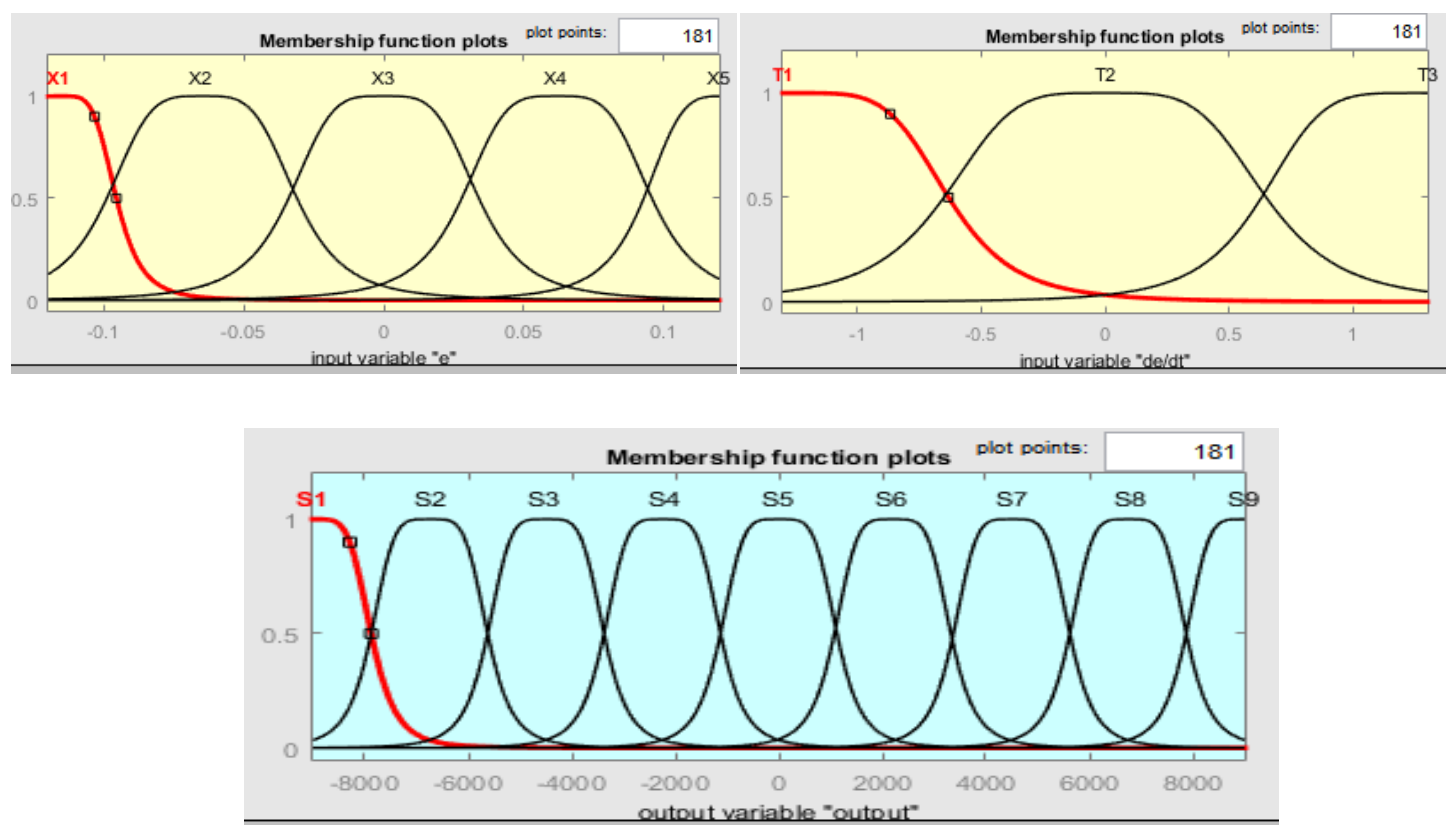

Şekil 2:

Bulanık Mantık Üyelik Fonksiyonları

Bu çalışmada bulanık mantık algoritması için genelleştirilmiş çan şekilli üyelik fonksiyonu (İngilizce kısaltılmışı gbellmf) kullanılmış olup, bunun matematik ifadesi şöyledir:

$$
f(x, a, b, c)=\frac{1}{1+\left|\frac{x-c}{a}\right|^{2 b}}
$$

Bu denklemde $a$, fonksiyonun yükselişe geçtiği nokta, $\mathrm{b}$; fonksiyonun yükselişteki orta noktası, c; fonksiyonun en yüksek noktasıdır. Bulanık mantık kural tablosu Tablo 2'deki gibi Güçlü (2005) tarafindan belirlenen değerlerden esinlenerek oluşturulmuştur.

Tablo 2. Bulanık Mantık Kural Tablosu

\begin{tabular}{|c|c|c|c|c|c|}
\hline de/dt-e & X1 & X2 & X3 & X4 & X5 \\
\hline T1 & S2 & S3 & S4 & S5 & S6 \\
\hline T2 & S3 & S4 & S5 & S6 & S7 \\
\hline T3 & S4 & S5 & S6 & S7 & S8 \\
\hline
\end{tabular}

Tablo 2'de verilen X, T ve S değişkenleri Şekil 2'de verilen bulanık mantık üyelik fonksiyonlarında gösterilmiştir. Doğrusal olmayan tam taşıt modeli için geliştirilen bulanık mantık tabanlı geri beslemeli kontrolcünün Matlab/Simulink şeması Şekil 3'te verilmiştir. Burada verilen kontrol sisteminde taşıtın dört köşesinde oluşan ve Denklem 1-4 ile verilen yer değiştirmeler geri beslenerek kontrol gerçekleştirilmiştir. 


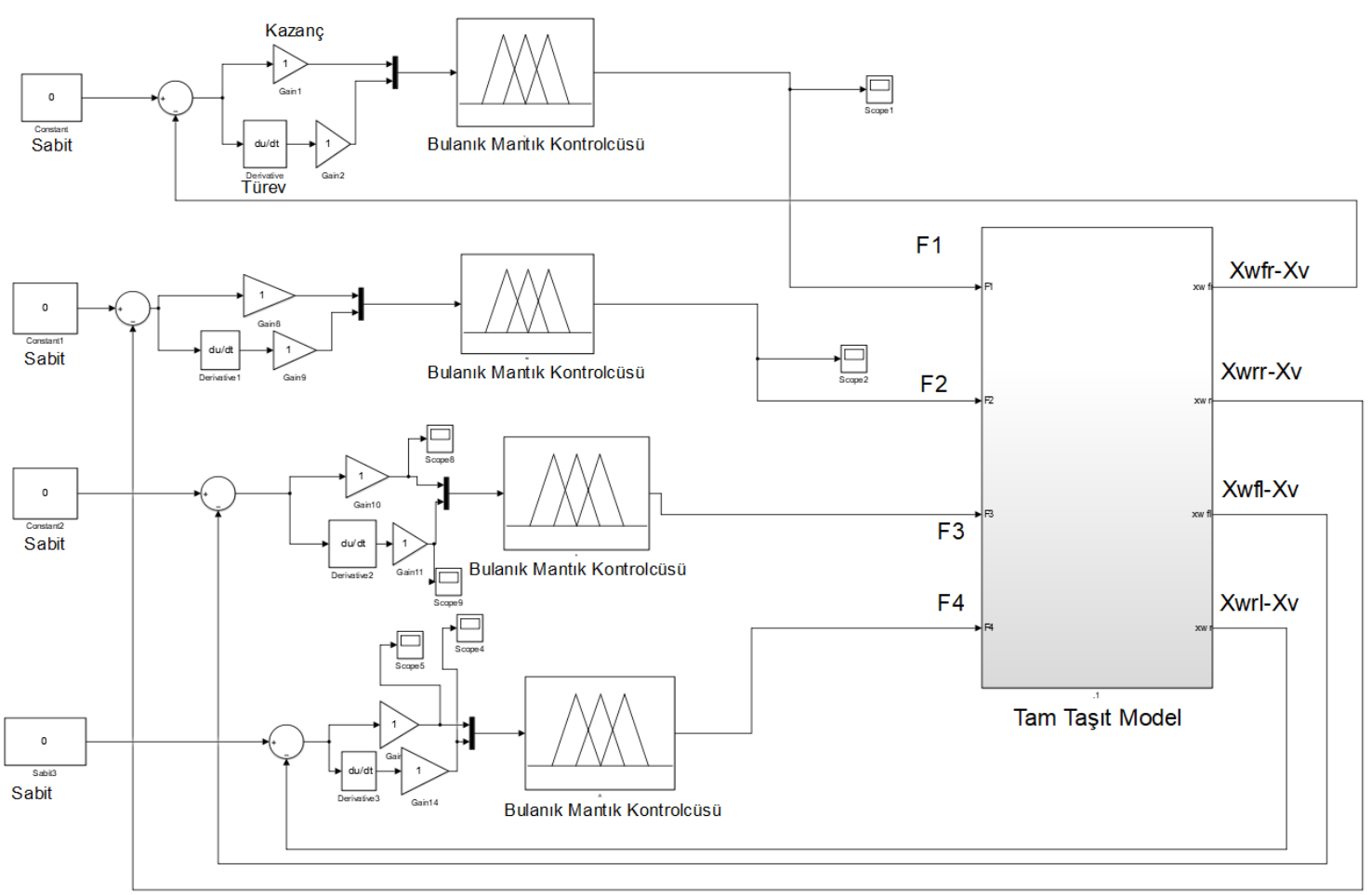

Şekil 3:

Bulanık mantık tabanlı geri beslemeli tam taşıt modeli kontrolü

\subsection{PID Tabanlı Kontrol}

Bir sistemde PID denetleyici ile sistemin giriş ve çıkış sinyalleri arasındaki fark geri beslenerek kontrol edilir. Bu işlemin yapılabilmesi için PID denetleyicinin davranışının ya da giriş çıkış fonksiyonları arasındaki ilişkinin bilinmesi gereklidir. Şekil 4'te birim geri beslemeli kontrol edilen bir sistemin blok diyagramı verilmiştir. 
Toksoy M.T., Yıldız A. : Tekerlek içi Mot. Elek. Arac. Akt. Süs. Sist. PID Bulanık Mant. Tab. Kontr.

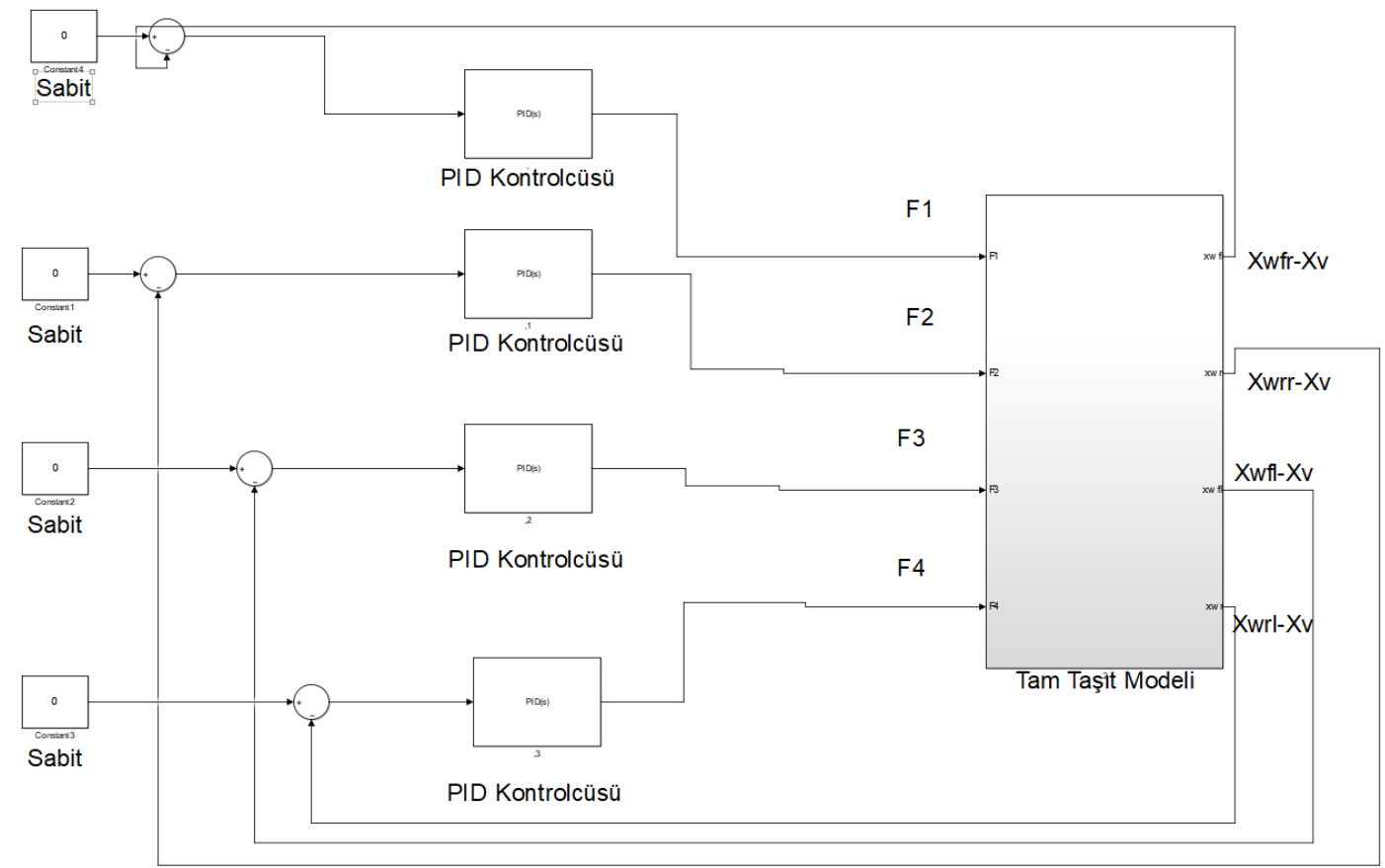

Sekil 4:

PID tabanlı Geri beslemeli tam taşıt modeli kontrolü

Bu sistemde PID kazanç katsayıları aşağıda belirtilen tablodan elde edilen değerler dikkate alınarak belirlenmiştir. Bunun için aşım ve oturma zamanı referans alınarak en iyi değerleri veren PID katsayıları seçilmiştir.

Tablo 3. $K_{p}$ belirleme tablosu

\begin{tabular}{|c|c|c|c|c|}
\hline $\boldsymbol{K}_{\boldsymbol{p}}$ & $\boldsymbol{K}_{\boldsymbol{i}}$ & $\boldsymbol{K}_{\boldsymbol{d}}$ & Aşım & $\begin{array}{c}\text { Oturma } \\
\text { Zaman1 }\end{array}$ \\
\hline $0-1000$ & 100 & 100 & $\% 52,46$ & 3,1568 \\
\hline $1000-5000$ & 100 & 100 & $\% 50,36$ & 3,0053 \\
\hline $5000-10000$ & 100 & 100 & $\% 45,63$ & 2,9124 \\
\hline $10000-20000$ & 100 & 100 & $\% 38,31$ & 2,8602 \\
\hline $20000-50000$ & 100 & 100 & $\% 33,27$ & 2,7516 \\
\hline $50000-60000$ & 100 & 100 & $\% 32,15$ & 2,7509 \\
\hline $60000-70000$ & 100 & 100 & $\% 37,22$ & 2,8573 \\
\hline
\end{tabular}


Tablo 4. $K_{i}$ belirleme tablosu

\begin{tabular}{|c|c|c|c|c|}
\hline $\boldsymbol{K}_{\boldsymbol{p}}$ & $\boldsymbol{K}_{\boldsymbol{i}}$ & $\boldsymbol{K}_{\boldsymbol{d}}$ & Aşım & $\begin{array}{c}\text { Oturma } \\
\text { Zaman1 }\end{array}$ \\
\hline 53600 & $0-20$ & 100 & $\% 34,46$ & 2,7953 \\
\hline 53600 & $20-50$ & 100 & $\% 34,34$ & 2,6215 \\
\hline 53600 & $50-90$ & 100 & $\% 33,86$ & 2,5326 \\
\hline 53600 & $90-140$ & 100 & $\% 32,03$ & 2,2217 \\
\hline 53600 & $140-200$ & 100 & $\% 33,25$ & 2,4632 \\
\hline 53600 & $200-500$ & 100 & $\% 34,85$ & 2,9135 \\
\hline
\end{tabular}

Tablo 5. $K_{d}$ belirleme tablosu

\begin{tabular}{|c|c|c|c|c|}
\hline $\boldsymbol{K}_{\boldsymbol{p}}$ & $\boldsymbol{K}_{\boldsymbol{i}}$ & $\boldsymbol{K}_{\boldsymbol{d}}$ & Aşım & $\begin{array}{c}\text { Oturma } \\
\text { Zaman1 }\end{array}$ \\
\hline 53600 & 136 & $0-100$ & $\% 32,79$ & 2,8327 \\
\hline 53600 & 136 & $100-500$ & $\% 32,34$ & 2,5215 \\
\hline 53600 & 136 & $500-1000$ & $\% 32,09$ & 2,4225 \\
\hline 53600 & 136 & $1000-1500$ & $\% 31,18$ & 2,4317 \\
\hline 53600 & 136 & $1500-200$ & $\% 31,86$ & 2,7666 \\
\hline
\end{tabular}

Burada amaç aşım oranını ve oturma zamanını optimize etmektir. Bu amaç doğrultusunda sistem için olabilecek en uygun değerler fiziksel sınırlara bağlı kalarak ve aşım ile oturma zamanını en minimal seviyeye çekebilecek biçimde oluşturulmuştur. Elde edilen en iyi değerler Tablo 3, Tablo 4 ve Tablo 5'de verilmiștir. Bu tabloların incelenmesinden ideal aşım yüzdesi ve ideal oturma zamanı değerleri sirasıyla \%31,10 ve 2,22 sn olarak bulunmuş olup bu değerlere tekabül eden kazanç katsayıları Tablo 6'da verilmiştir.

Tablo 6. PID kazanç katsayıları değer tablosu

\begin{tabular}{|c|c|}
\hline Katsayılar & Değerler \\
\hline $\boldsymbol{K}_{\boldsymbol{p}}$ & 53600 \\
\hline $\boldsymbol{K}_{\boldsymbol{i}}$ & 136 \\
\hline $\boldsymbol{K}_{\boldsymbol{d}}$ & 1008 \\
\hline
\end{tabular}

\section{SIMULASYON SONUÇLARI}

Bu bölümde yukarıda verilen PID ve bulanık mantık değerleri kullanılarak sistemin zaman cevabına ait simülasyon sonuçları, Hareket denklemlerinin dördüncü mertebeden Runge_Kutta yöntemiyle sayısal çözümünden elde edilmiştir. Grafiklerde kontrolcü kullanılması ve kullanılmaması haline ait eğriler mukayese amacıyla bir arada verilmiştir. 
Toksoy M.T., Yıldız A. : Tekerlek içi Mot. Elek. Arac. Akt. Süs. Sist. PID Bulanık Mant. Tab. Kontr.

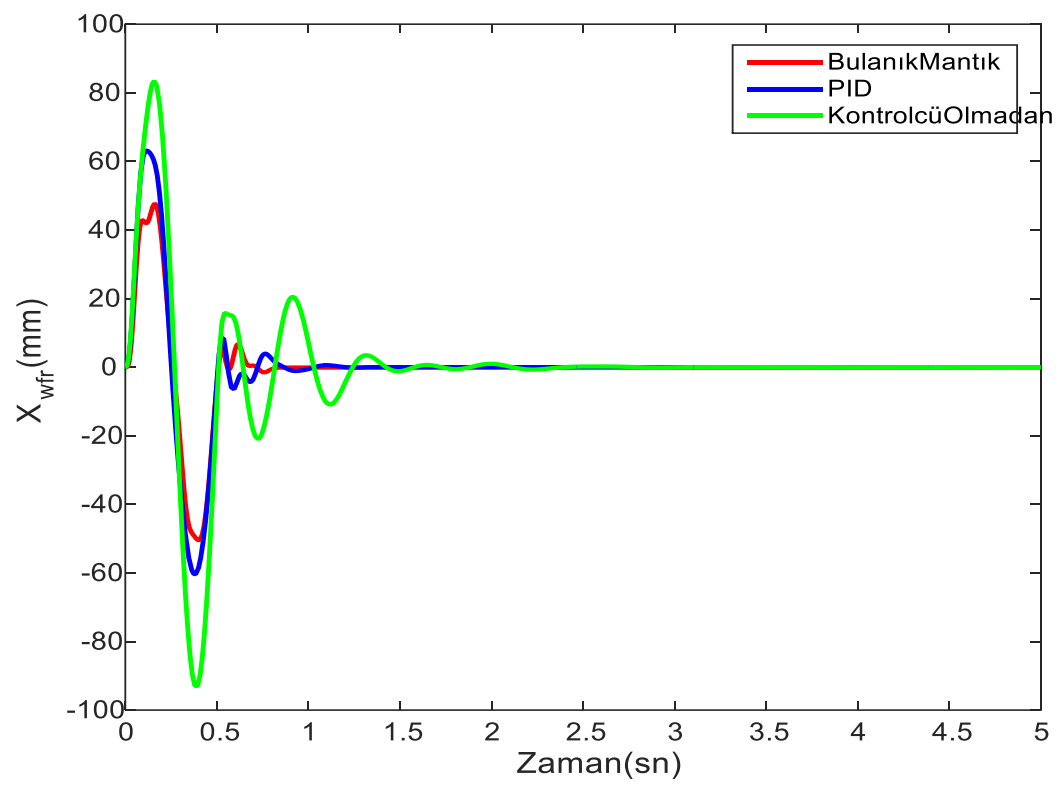

Şekil 5:

Să̆ ön teker titreşim grafiği

Sağ ve sol ön teker ile arka teker titreşim grafikleri sırasıyla Şekil 5, Şekil 6, Şekil 7 ve Şekil 8'de verilmiştir. Taşıtın koltuk, ana gövde ve başvurma hareketlerinin grafikleri de sırasıyla Şekil 9, Şekil 10 ve Şekil 11'de verilmiştir. Bu grafiklerden görüldüğü üzere en düşük titreşim genliği Bulanık Mantık tabanlı denetleyicisi daha sonra PID kontrolcü ile elde edilmiştir. Kontrolcü olmadan pasif süspansiyon sisteminde en büyük titreşim genlikleri gözlemlenmiştir.

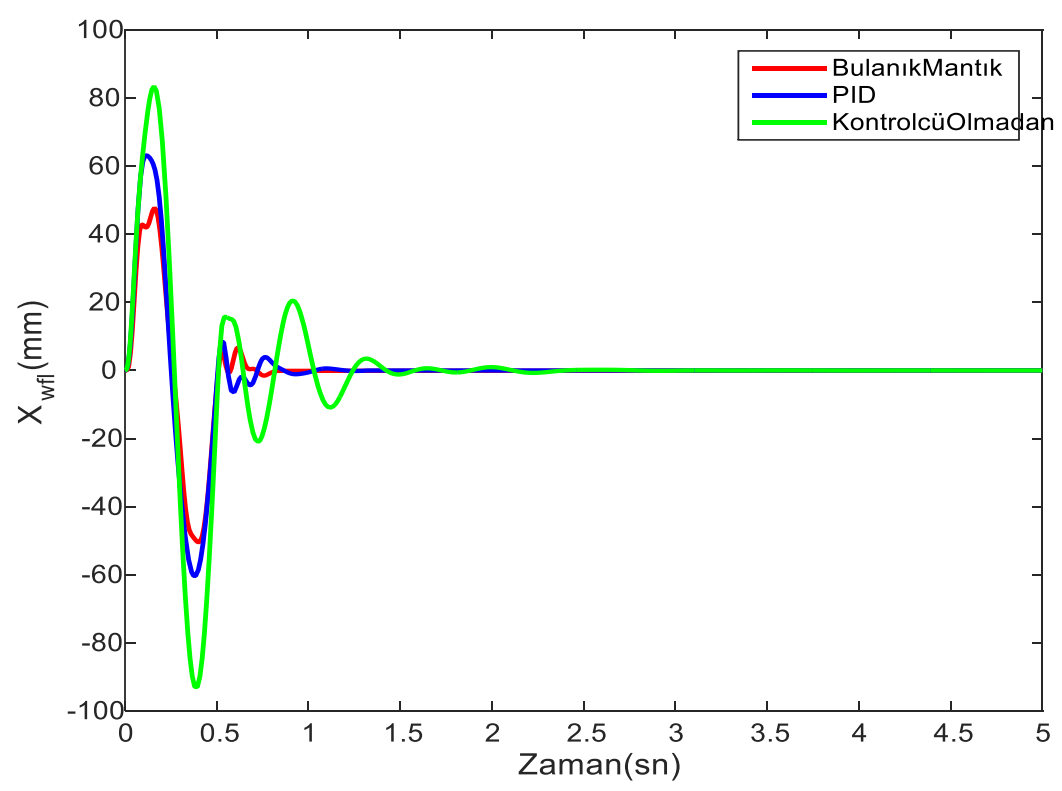

Şekil 6:

Sol ön teker titreşim grafiği 


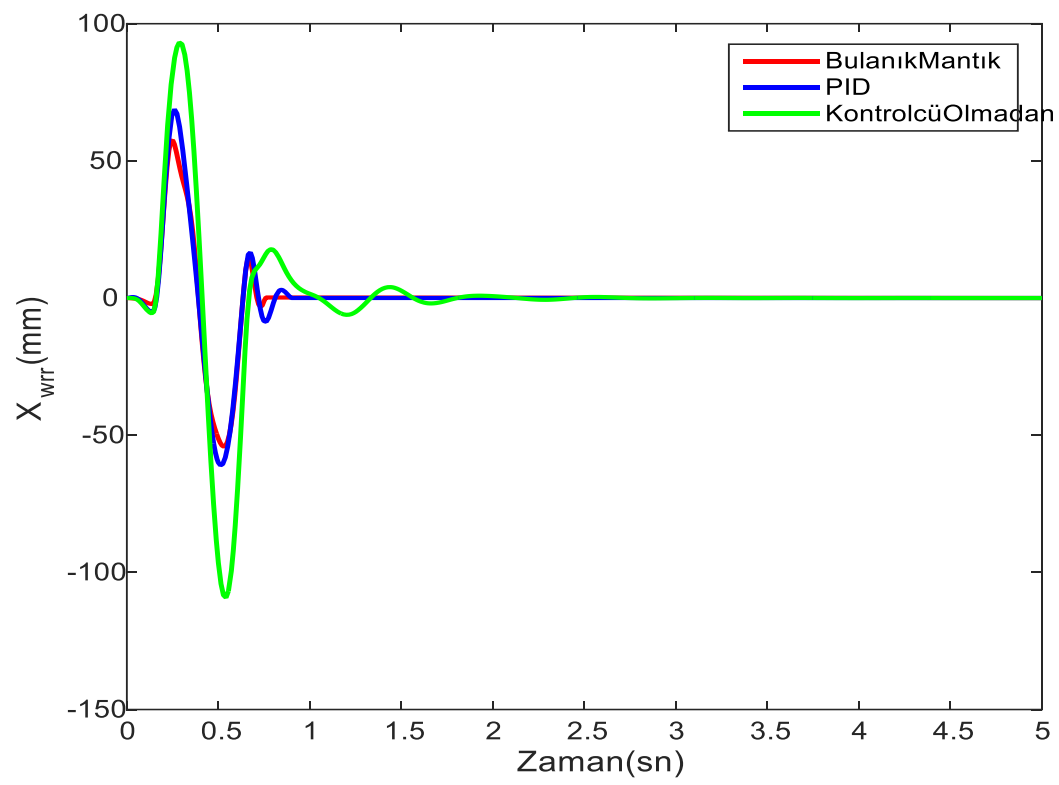

SSekil 7:

Să̆ arka teker titreşim grafiğ $i$

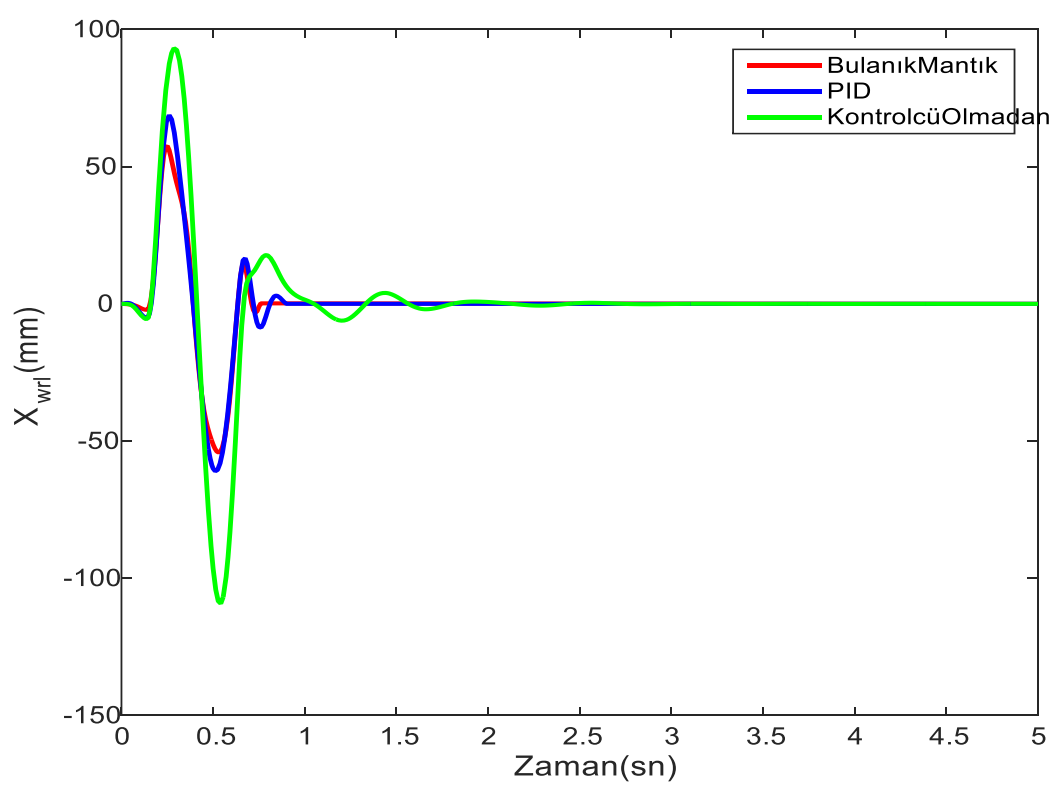

Şekil 8:

Sol arka teker titreşim grafiği 
Toksoy M.T., Yıldız A. : Tekerlek içi Mot. Elek. Arac. Akt. Süs. Sist. PID Bulanık Mant. Tab. Kontr.

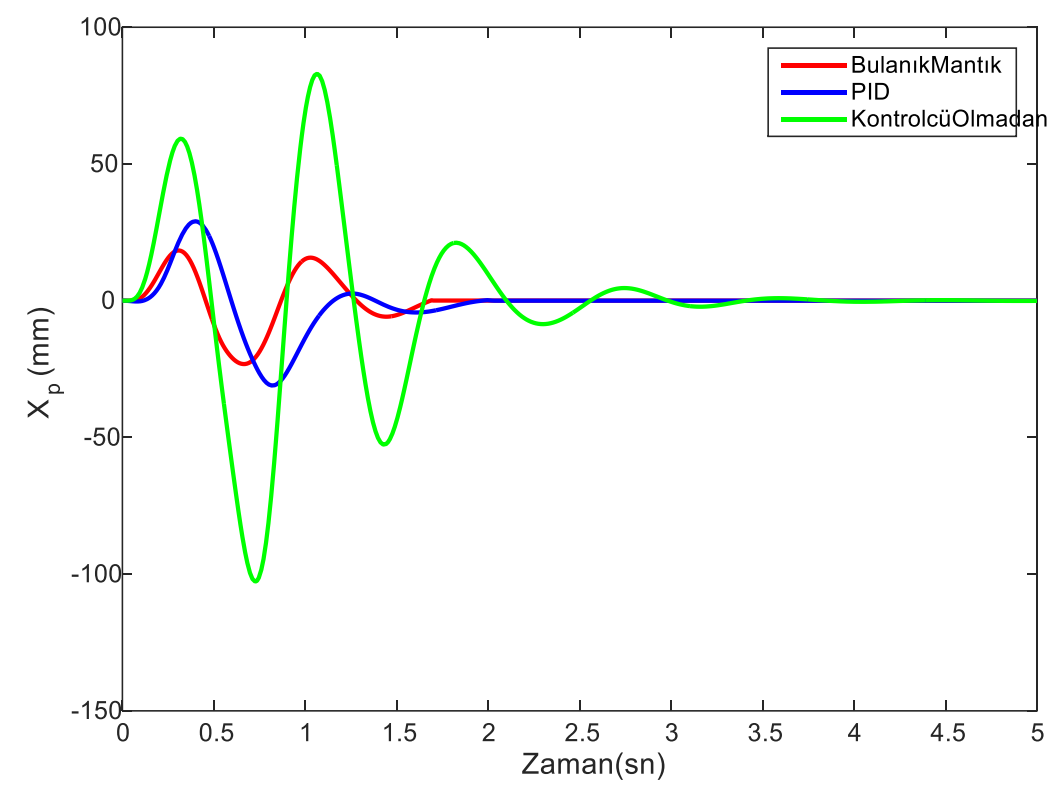

Şekil 9:

Taşıtın koltuk titreşim grafiğ $i$

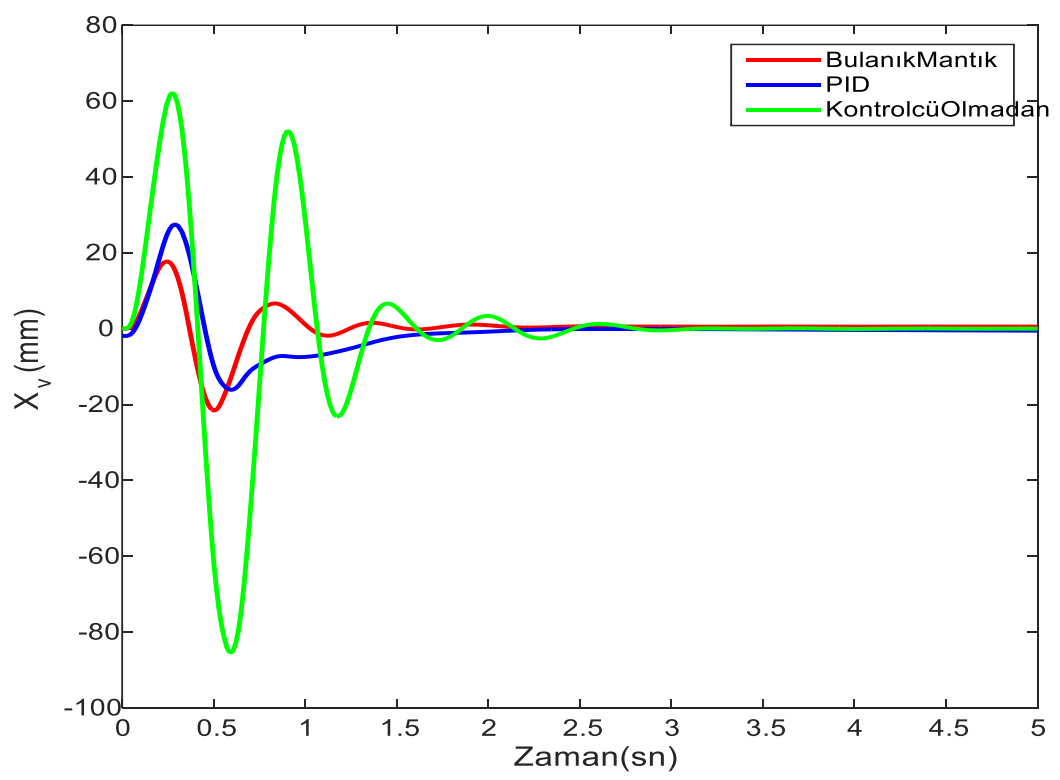

Şekil 10:

Taşıt gövdesinin titreşim grafiği 


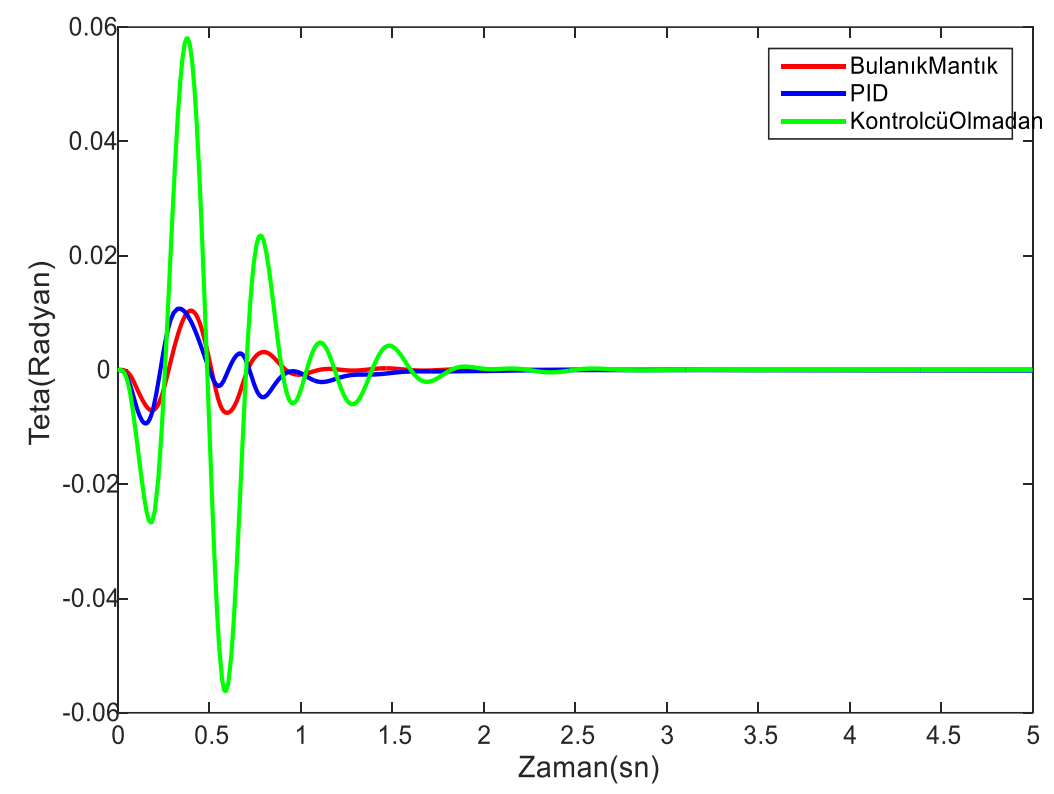

Şekil 11:

Taşıt başvurma hareketi titreşim grafiği

\section{DEĞERLENDİRME}

$\mathrm{Bu}$ çalışma arkadan itişli ve tekerlek içi motorlu elektrikli bir aracın matematiksel modellenmesi ile aktif süspansiyon sistemlerinin farklı yöntemlerle karşılaştırmalı kontrolü incelenmiştir. Bunun için bulanık mantık ve PID tabanlı Matlab/Simulink ortamında hareket denklemleri yazılmış ve yukarıda anlatıldığı şekilde aracın titreşim hareketleri kontrol edilmiştir. Elde edilen sayısal sonuçlar aşım değeri ve oturma zamanı açısından bulanık mantık kontrol algoritmasının PID kontrole göre daha etkin olduğunu ortaya koymaktadır. Ayrıca kontrolcü olmadan yapılan testte genliklerin ve oturma zamanının oldukça yüksek olduğu gözlemlenmiştir. Gelecek çalışmalarda daha hızlı ve etkin bir kontrol gerçekleştirebilmek için PID ve bulanık mantık denetleyicilerinin değerleri güncel ve efektif bir optimizasyon yöntemi ile tespit edilmesi planlanmıştır.

\section{KAYNAKLAR}

1. Allen, J.A. (2008) Design of active suspension control based upon use of tubular linear motor and quarter-car model, Master Thesis, Texas A\&M University, Texas.

2. Badran, S., Salah, A., Abbas, W., Abouelatta, O.B. (2012) Design of Optimal Linear Suspension for Quarter Car with Human Model using Genetic Algorithms, The Research Bulletin of Jordan ACM, 2(2), 42-51.

3. Cao, D., Song, X., Ahmadian, M. (2011) Editors' perspectives: road vehicle suspension design, dynamics, and control, Vehicle System Dynamics, 49(1-2), 3-28. doi:

10.1080/00423114.2010.532223 
Toksoy M.T., Yıldız A. : Tekerlek içi Mot. Elek. Arac. Akt. Süs. Sist. PID Bulanık Mant. Tab. Kontr.

4. Doğan, H., Kaplan, K., Kuncan, M., Ertunç, H.M. (2015) Araç Süspansiyon Sistemi Kontrolüne PID ve Bulanık Mantık Yaklaşımları, Otomatik Kontrol Ulusal Toplantısı, Denizli, 699-704.

5. Gillespie, T.D. (1996) Fundamentals of Vehicle Dynamics, SAE International, Warrendale.

6. Güçlü, R. (2005) Fuzzy Logic Control of Seat Vibrations of a Non-Linear Full Vehicle Model, Nonlinear Dynamics, 40(2005), 21-34. doi: 10.1007/s11071-005-3815-7

7. Jazar, R. N. (2017) Vehicle Dynamics: Theory and Application, Springer International, New York.

8. Jin, L., Yu, Y., Fu, Y. (2016) Study on the ride comfort of vehicles driven by in-wheel motors, Advanced Mechanical Engineering, 8(3), 1-9. doi: 10.1177/1687814016633622

9. Li, H., Jing, X., Karimi, H.R. (2014) Output-feedback-based $\mathrm{H}_{\infty}$ control for vehicle suspension systems with control delay, IEEE Transactions on Industry Applications, 61(1), 436-46. doi: 10.1109/TIE.2013.2242418

10. Mehdizadeh, S. A. (2015) Optimization of passive tractor cabin suspension system using ES, PSO and BA, Journal of Agricultural Technology, 11(3), 595-607.

11. Nikam, S., Vandana, R., Fernandes, B. A. (2012) High-Torque-Density Permanent-Magnet Free Motor for In-Wheel Electric Vehicle Application, IEEE Transactions on Industry Applications, 48(6), 2287-2295. doi: 10.1109/TIA.2012.2227053

12. Oliveira, K., Cesar, M., Goncalves, J. (2017) Fuzzy based Control of a Vehicle Suspension System using a MR Damper, Proceedings of the 12th Portuguese Conference on Automatic Control, Guimaraes, 14-16.

13. Özkop, E., Altaş, İ.H. (2007) Bulanık Mantık Denetleyici ile Aktif Otomobil Süspansiyon Denetimi, 12. Elektrik Elektronik Bilgisayar Biyomedikal Mühendisliği Ulusal Kongresi ve Fuarı, Eskişehir, 14-18.

14. Rao, M.V.C., Prahlad, V. (1997) A tunable fuzzy logic controller for vehicle-active suspension systems, Fuzzy Sets and Systems, 85(1), 11-21. doi: 10.1016/01650114(95)00369-X

15. Shirahatt, A., Prasad, P.S.S., Panzade, P., Kulkarni, M.M. (2008) Optimal Design of Passenger Car Suspension for Ride and Road Holding, Journal of the Brazilian Society of Mechanical Sciences and Engineering, 30(1), 67-68. doi: 10.1590/S167858782008000100010

16. Sun, W., Pan, H., Yu, J., Gao, H. (2014) Reliability control for uncertain half-car active suspension systems with possible actuator faults, IET Control Theory \& Applications, 8(9), 746-754. doi: 10.1049/iet-cta.2013.0471

17. Takagi, T., Sugeno, M. (1985) Fuzzy identification of systems and its applications to modeling and control, IEEE Transactions on Systems, Man, and Cybernetics, 15(1), 116-132. doi: 10.1109/TSMC.1985.6313399

18. Takahashi, T., Takemoto, M., Ogasawara, S., Hino, W., Takezaki, K.S. (2017) Size and weight Reduction of an In-wheel Axial-gap Motor Using Ferrite Permanent Magnets for Electric Commuter Cars, IEEE Transactions on Industry Applications, 53(4), 3927-3935. doi: 10.1109/TIA.2017.2684739.

19. Yokoyama, M., Hedrick, J.K., and Toyama, S. (2001) A model following sliding mode controller for semi-active suspension systems with MR dampers. Proceedings of the American Control Conference, Arlington, 25-27. 
20. Yildiz, A. (2019a) Optimum suspension design for non-linear half vehicle model using particle swarm optimization (PSO) algorithm, The 41st International JVE Conference Vibration, Leipzig, 43-48. doi: 10.21595/vp.2019.21012

21. Y1ldiz, A. (2019b) A comparative study on the optimal non-linear seat and suspension design for an electric vehicle using different population-based optimisation algorithms, International Journal of Vehicle Design, 80(2/3/4), 241-256, doi: 10.1504/IJVD.2019.10031168. 
